Published in final edited form as:

Transfusion. 1985 ; 25(2): 120-123.

\title{
Blood transfusion in liver transplantation
}

\author{
Patricia Butler, MT (ASCP) SBB [Supervisor], \\ Transfusion Service, Central Blood Bank \\ Linda Israel, MT (ASCP) SBB [Director], \\ Patient Laboratory Services, Central Blood Bank
}

Jacob Nusbacher, MD [Executive Vice President and Medical Director],

Central Blood Bank of Pittsburgh, 811 Fifth Avenue, Pittsburgh, PA 15219

David E. Jenkins Jr., MD [President], and

Central Blood Bank

Thomas E. Starzl [Professor of Surgery]

University of Pittsburgh, School of Medicine, Pittsburgh, P A

\begin{abstract}
Liver transplantation is a relatively new procedure in which unusually large quantities of blood are used. Blood use in 68 adult and 49 pediatric liver transplants was reviewed. The median (range) intraoperative red cell use for adults and children was 28.5 (3-251) and 11 (255), respectively. Blood use closely correlated with the patient's primary diagnosis. Adult patients with primary biliary cirrhosis and carcinoma used about one-half as much blood as those with a diagnosis of sclerosing cholangitis, hepatitis, or cirrhosis. Patients in the former diagnostic groups also had better survival rates. Total red cell use for the patient's entire hospitalization was about twice that used during surgery. Fresh-frozen plasma use paralleled red cell transfusions, but platelet use was modest. These data can serve as a baseline in helping other hospital transfusion services prepare for the advent of liver transplantation in their institutions.
\end{abstract}

Until recently, liver transplantation was performed infrequently and in only a few hospitals in this country. Among the factors contributing to the paucity of liver transplants have been the lack of available donor organs, the difficulty of the surgical procedure, the inability to store donor livers after harvesting, the inability to perform histocompatibility testing prior to transplantation within the necessary time constraints, and the relatively high organ rejection rates. However, with improved surgical technics, increased public awareness of the need for organs, and improved immunosuppression, liver transplantation is now being introduced in more hospitals.

Liver transplantation also is associated with the use of large volumes of blood, considerably more than with most other surgical procedures. This communication reviews the blood transfusion needs in a large number of adult and pediatric patients undergoing liver transplantation in our institution over a 2-year period.

\section{Materials and Methods}

Between February 1981 and April 1983, 68 liver transplantations were performed on adults at Presbyterian-University Hospital and 49 were performed on children at Children's Hospital of 
Pittsburgh. All transplantations were performed with cyclosporine immunosuppression. The quantity of red cells (RBCs), fresh-frozen plasma (FFP), and platelets transfused was ascertained. Intraoperative and perioperative transfusions were recorded separately. Transfusion needs were determined by the transplant surgical team or other physicians caring for the patient.

All RBC transfusions were in the form of red cells ("packed cells"). No special attention was given to the age of units distributed for transfusion. ABO-matched units were given unless inventory needs mandated the use of $\mathrm{ABO}$-compatible, but not specific, units. Rh-negative recipients were given Rh-negative units early during surgery, but if large amounts of blood were needed, Rh-positive units were given routinely. FFP and platelet transfusions were given on the basis of clinical need as ascertained by the transplant team.

In 61 of the 68 adult transplant patients, the diagnosis fit into one of the following four categories: 1) primary biliary cirrhosis, 2) carcinoma, 3) sclerosing cholangitis, and 4) hepatitis or cirrhosis. Among the 49 pediatric transplant patients, 37 could be categorized into one of the following four diagnostic categories: 1) biliary atresia, 2) alpha-1-antitrypsin deficiency, 3) hepatitis or cirrhosis, and 4) carcinoma.

Of the 68 adult transplant procedures, 15 were retrans-plantations-a second liver transplant occurring after failure of the initial graft. Among the pediatric patients, there were 19 second transplantations in the initial 49 procedures analyzed. Survival was defined as discharge from the hospital.

\section{Results}

\section{Adult procedures}

A frequency distribution of intraoperative RBC use is illustrated in Fig. 1. Also shown is the mortality (as defined above) as a function of the number of units transfused. In more than onehalf of all procedures ( 38 of 68), less than 30 units of blood were transfused. Figure 1 also illustrates the strong correlation between units transfused and patient outcome. The survival rate was 60 percent when less than 30 units were transfused, but only 33 percent when more than 30 units were given. Only 5 of 15 patients survived after retransplantation procedures.

The use of FFP paralleled red cell use in every aspect (data not shown). There was a virtual one-to-one relationship between FFP nd RBC infusions. The intraoperative use of platelets is illustrated in Figure 2. In 43 of 68 procedures (63\%), less than 20 units of platelets were given. In 14 procedures (20\%), no platelets were used. Intraoperative blood use correlated strongly with the patient's primary diagnosis. As shown in Table 1, patients with a diagnosis of primary biliary cirrhosis (PBC) or carcinoma used less than one-half the quantity of RBCs required for patients with sclerosing cholangitis, hepatitis, or cirrhosis. Only three of 16 patients with PBC and two of nine patients with carcinoma used more than 30 units of RBCs. Survival rates in these groups of patients were substantially better than those in patients with other diagnoses.

\section{Pediatric procedures}

A frequency distribution of intraoperative RBC use in children is illustrated in Figure 3. The data are similar to those observed in adults (Fig. 1), although the overall quantity of blood transfused was considerably less. In 32 of 49 (65\%) procedures, less than 15 units of RBCs were given.

However, overall survival correlated less well with blood use in children. There was a 65 percent survival rate when less than 15 units of RBCs were transfused and a 58 percent survival 
rate when more than 15 units of RBCs were transfused. Among pediatric patients who underwent a second transplantation procedure, the survival rate was 47 percent ( 9 of 19).

As in adult patients, children with a diagnosis of hepatitis or cirrhosis required considerably more RBC transfusions - nearly twice as many — as those with other conditions (Table 2). The survival rate in children also correlated with blood use when the data were grouped by diagnoses.

\section{Total blood component use}

Table 3 summarizes the total blood component use for both adults and children during the entire hospitalization for liver transplantation. The total number of units of RBCs, FFP, and platelets before and after surgery was approximately equivalent to that given intraoperatively. Most perioperative transfusion events, however, were not massive, but consisted of a few units given during the long pre- and postoperative hospitalizations common in these patients.

\section{Discussion}

Liver transplantation makes unusual demands on the blood bank. The total use of red cell products in this group of patients (both intra- and perioperative) accounted for about 15 percent of all transfusion needs in our institution and about 4.7 percent of the 130,000 units of blood distributed annually to all hospitals served by the Central Blood Bank of Pittsburgh. More than one-half of these transfusions occurred intra-operatively, a fact that clearly indicates the need for logistic planning by the blood bank in support of these procedures.

Liver transplants occur with little notice. The maximal time between donor organ harvest and transplantation is less than twelve hours, often considerably less. Because of their technical difficulty, these procedures take from 6 to 24 hours to perform and are often done at night or on weekends. All these factors tend to stress the transfusion service's capability to support these patients.

In our experience, a key to successful transfusion support in liver transplantation is good communication between the transplant team and the transfusion service. The transfusion service must be notified immediately when an organ becomes available and the recipient is selected. This permits accumulation of red cell and other transfusion products of the proper type and in the necessary amount. Good communication is also important during surgery. The occurrence of unexpected bleeding or technical problems should be relayed immediately to the transfusion service so that adequate quantities of blood can be prepared in advance.

The magnitude of bleeding during liver transplantation appears to result from two factors: the poor hemostatic capabilities of many of these patients and the technical difficulty of the surgical procedure. The former problem is the subject of another communication. I The latter, as indicated in Tables 1 and 2, is a major factor in determining not only blood use but also patient survival (Figs. 1 and 3). Patients with sclerosing cholangitis and hepatitis or cirrhosis usually have severe portal hypertension which results in the proliferation and engorgement of collateral blood vessels which tend to bleed profusely during surgery. Furthermore, these patients often have had previous operations which tends to make surgical dissection and vascular anastamoses much more difficult. In these cases, much of the intraoperative bleeding occurs early in the procedure, during the removal of the recipient's diseased liver and during the anhepatic period. In contrast, patients with primary biliary cirrhosis or carcinoma often have "cleaner" surgical fields and the dissection proceeds with greater ease. These patients use less blood and have a better prognosis. 
These observations can form the basis for readying the transfusion service for liver transplantation procedures. Adult patients with primary biliary cirrhosis or carcinoma need have only 20 units available with 20 held in reserve. Other patients will need about twice this number. A similar strategy can be developed for pediatric recipients. In our view, standard compatibility tests need be done for only the first 10 to 20 units transfused. Rechecking the donor's $\mathrm{ABO}$ type (e.g., immediate spin) is all that is required thereafter.

When the transplant recipient has a clinically significant alloantibody, additional planning is required. A satisfactory approach has been to use blood negative for the antibody specificity for the first 10 to 20 units. If substantial bleeding continues, any available ABO-compatible blood can be used until close to the end of the procedure. Administration of antigen-negative blood is then resumed for the estimated final 10 to 20 units. This approach has been used in a number of our transplant patients - in a patient with anti-c and anti-E or anti-D for examplewith no ill effects or delayed hemolytic transfusion reactions. However, it also demands excellent communication with the surgical team during the operation.

The large amount of FFP used in our patients reflects, in pan, the poor hemostatic capabilities of many of the liver transplant recipients. Bontempo et al. ${ }^{1}$ showed a correlation between the number of coagulation abnormalities prior to liver transplantation and the quantity of red cells transfused during the procedure. In addition, little whole blood is available in our region because the overall demand for components is so great. In our opinion, the use of whole blood, or a combination of whole blood and packed cells supplemented by either occasional FFP, single donor plasma, or cryoprecipitate would be an equally effective approach.

The use of intraoperative autologous transfusion in liver transplantation is desirable because it is possible that the blood bank's inventory could be depleted by this procedure. We have used this technic (Cell Saver, Haemonetics Corp, Braintree, MA) in a few patients. We found that the equipment could not handle effectively the large volume of blood presented to it; therefore, the surgeon had to switch back and forth between the standard suction apparatus and suction destined for reinfusion. This relatively minor problem could be solved by making minor alterations in the suction equipment, using more than one autologous infusion set, or by the development of faster autologous infusion equipment.

\section{Acknowledgments}

The authors thank Glenn Ramsey, MD, for his useful comments and Ruby Love for excellent assistance. We are also grateful to Judi Sherwin, MT(ASCP)SBB, Georgianne Hartwell MT(ASCP)SBB, and the donor resources and product management departments of Central Blood Bank for their efforts in supporting the liver transplantation program.

\section{References}

1. Bontempo FA, Lewis JH, Yan Thiel D, Spero JA, Ragni MV, Butler P, et al. The relation of preoperative coagulation findings to diagnosis, blood usage and survival in adult liver transplant. Transplantation. (in press). 


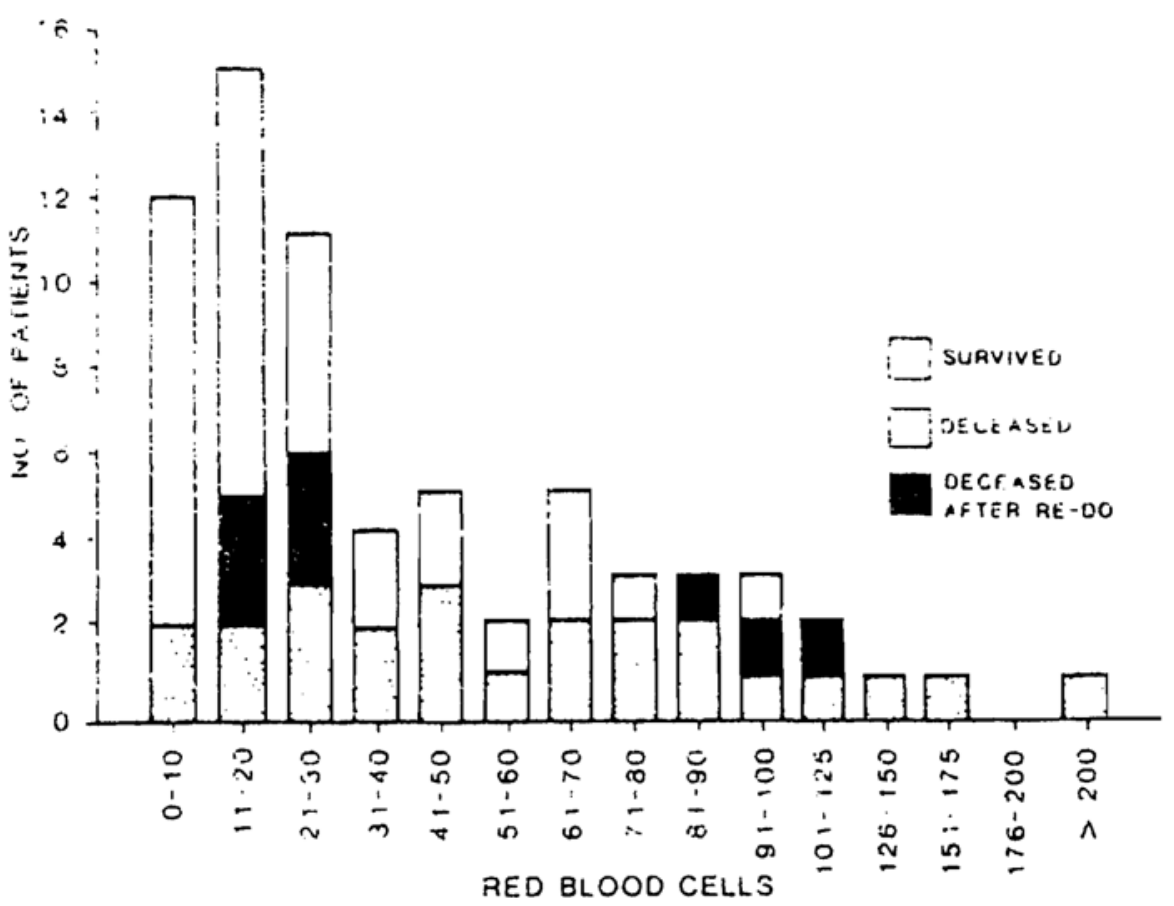

Fig. 1.

Intraoperative $\mathrm{RBC}$ use for 68 adult liver transplantations procedures. 


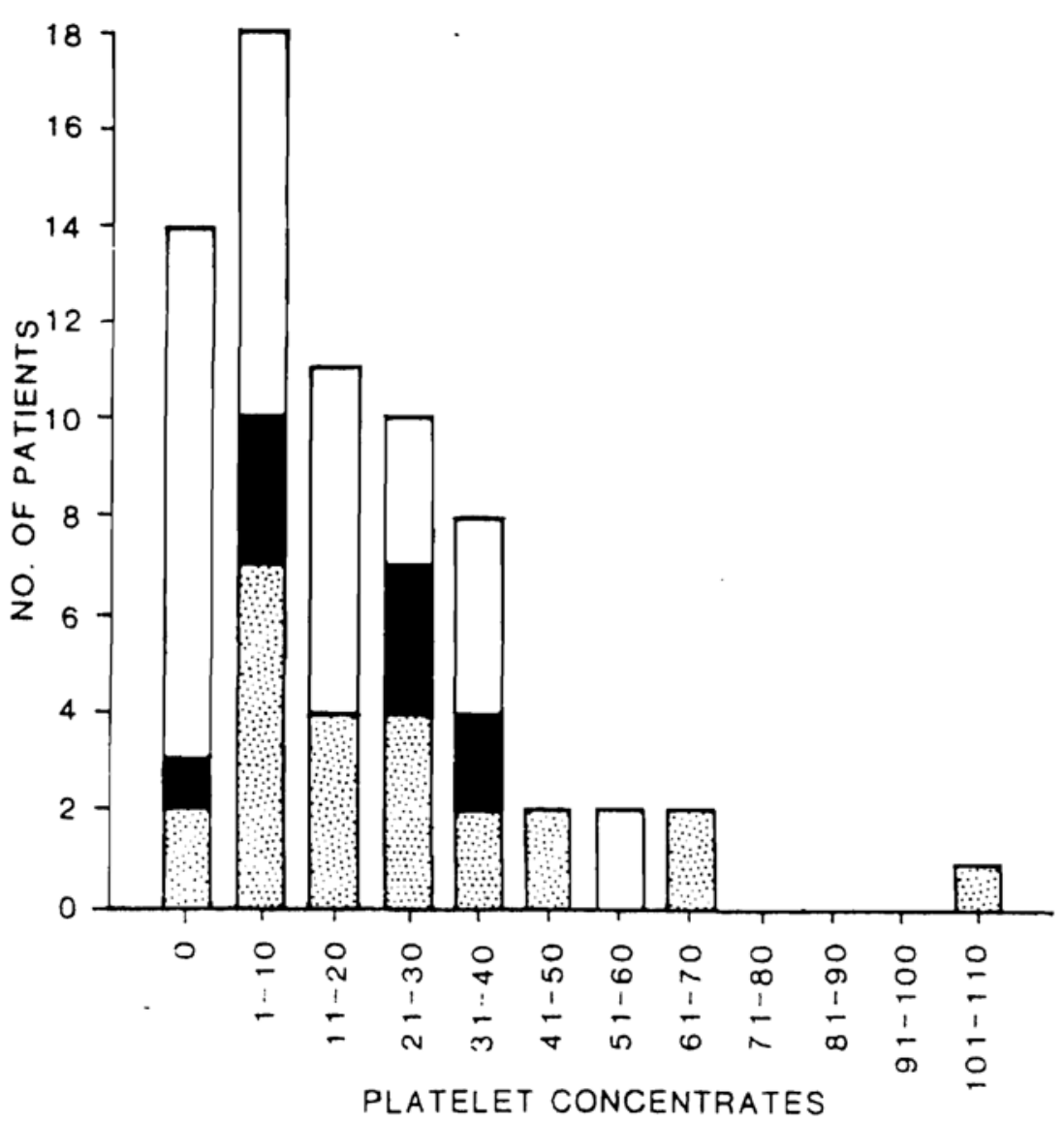

Fig. 2.

Intraoperative platelet use for 68 adult liver transplantation procedures (See Fig. 1 for legend). 


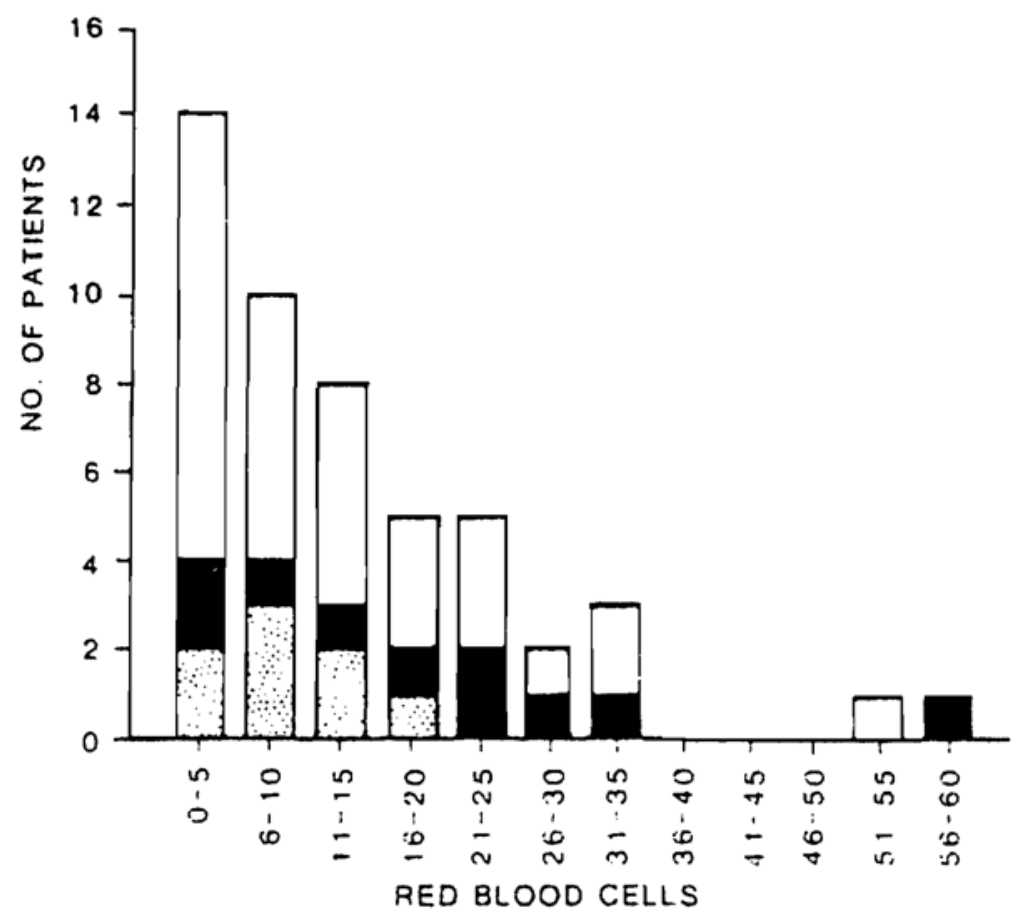

Fig. 3.

Intraoperative RBC use for 49 pediatric liver transplantation procedures (See Fig. 1 for legend). 
Table 1

RBC use in adult liver transplantation procedures correiated with patient diagnosis and survival

\begin{tabular}{lrlc}
\hline Diagnosis & Number of Patients & Intraoperative Median (Range) & Percent Survival \\
\hline Primary biliary Cirrhosis & 16 & $15(3-123)$ & 69 \\
Carcinoma & 9 & $17(6-65)$ & 67 \\
Sclerosing cholangitis & 8 & $36(10-143)$ & 50 \\
Hepatitis or cirrhosis & 28 & $3917-251)$ & 46 \\
\hline
\end{tabular}


Table 2

RBC use in pediatric liver transplantation procedures correlated with patient diagnosis and survival

\begin{tabular}{lrrr}
\hline Diagnosis & Number of Patients & Intraoperative Median (Range) & Percent Survival \\
\hline Biliary atresia & 20 & $10(3-55)$ & 65 \\
Alpha-1-antitrypsin deficiency & 8 & $8(2-21)$ & 75 \\
Hepatitis or cirrhosis & 6 & $19(5-23)$ & 50 \\
Carcinoma & 3 & $10(5-15)$ & 100 \\
\hline
\end{tabular}




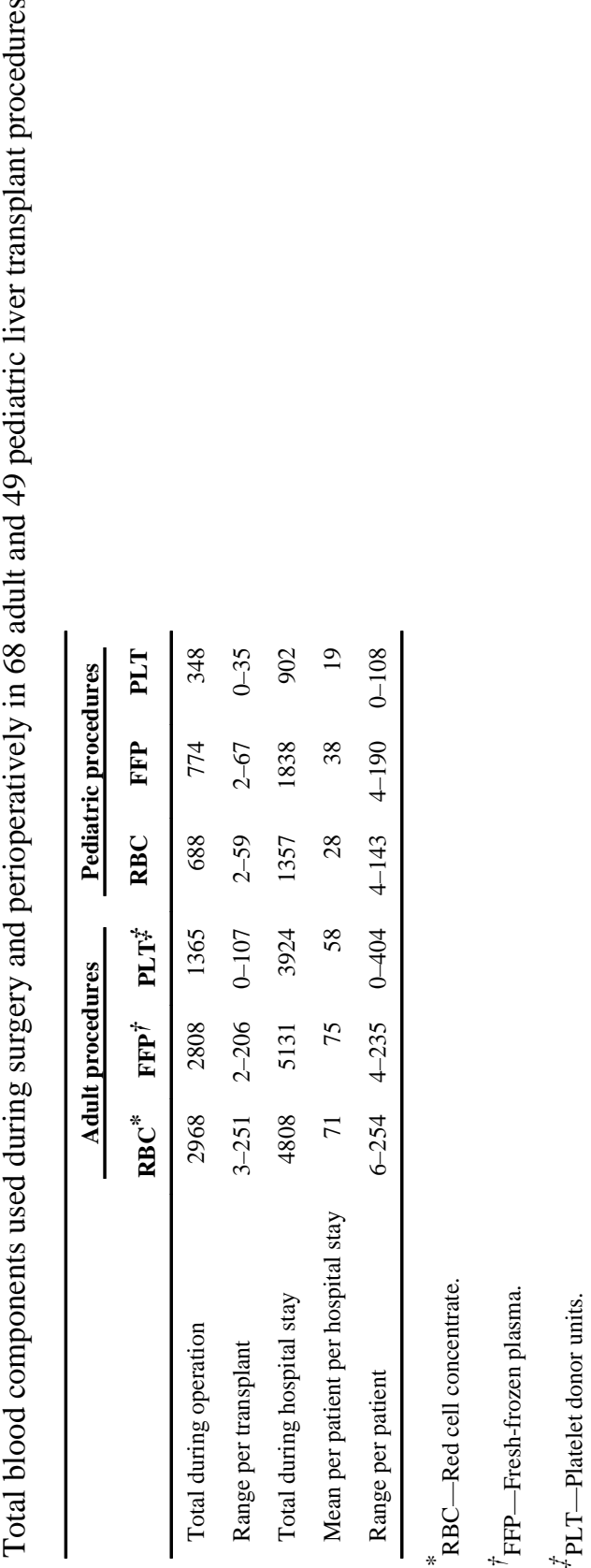

